\title{
Update on systemic treatment of upper tract urothelial carcinoma: a narrative review of the literature
}

\author{
Kilian M. Gust ${ }^{1}$, Irene Resch $^{1}$, David D'Andrea ${ }^{1}$, Shahrokh F. Shariat ${ }^{1,2,3,4,5,6}$ \\ ${ }^{1}$ Department of Urology, Comprehensive Cancer Center, Medical University of Vienna, Vienna, Austria; ${ }^{2}$ Department of Urology, Weill Cornell \\ Medical College, New York, NY, USA; ${ }^{3}$ Department of Urology, University of Texas Southwestern, Dallas, TX, USA; ${ }^{4}$ Department of Urology, \\ Second Faculty of Medicine, Charles University, Prague, Czech Republic; ${ }^{5}$ Institute for Urology and Reproductive Health, I.M. Sechenov First \\ Moscow State Medical University, Moscow, Russia; ${ }^{\circ}$ Department of Special Surgery, University of Jordan, Amman, Jordan \\ Contributions: (I) Conception and design: KM Gust, SF Shariat; (II) Administrative support: None; (III) Provision of study materials or patients: KM \\ Gust; (IV) Collection and assembly of data: KM Gust, I Resch, SF Shariat; (V) Data analysis and interpretation: KM Gust, D D'Andrea, SF Shariat; (VI) \\ Manuscript writing: All authors; (VII) Final approval of manuscript: All authors. \\ Correspondence to: Kilian M. Gust. Department of Urology, Medical University of Vienna, Vienna General Hospital, Waehringer Guertel 18-20, 1090 \\ Vienna, Austria. Email: kilian.gust@meduniwien.ac.at.
}

\begin{abstract}
Urothelial cancer (UC) is most commonly found in the urinary bladder, but can also appear in the upper urinary tract, where it is associated with several disease-specific challenges affecting its diagnosis, clinical staging, surgical management, and systemic therapy. A significant number of patients experience extra-vesical disease recurrence despite radical nephroureterectomy (RNU), leading to inevitable demise. Over the last years, the therapeutic armamentarium of UC has expanded with several systemic treatment options entering clinical care and deliver the potential to support a more individualized treatment in the near future. Currently, novel targeted therapies are emerging, accompanied with extensive biomarker research, which leads to a better understanding of the disease and therefore, reshaping the treatment landscape continuously and decisively. Though, systemic treatment of UTUC comes along with certain challenges that are specific to the disease, e.g., loss of renal function after RNU, which might result in ineligibility for a cisplatin-based chemotherapy. In this narrative review, the current standard of systemic treatment of UC in the perioperative and metastatic treatment setting are reported, with focus on UTUC. In addition, molecular aspects of UTUC, as well as future directions and specific implications for treatment of patients diagnosed with UTUC are discussed.
\end{abstract}

Keywords: Systemic therapy; chemotherapy; immune-checkpoint inhibitor; urothelial cancer; upper tract urothelial carcinoma (UTUC)

Submitted Jan 26, 2021. Accepted for publication Aug 26, 2021.

doi: $10.21037 /$ tau-21-47

View this article at: https://dx.doi.org/10.21037/tau-21-47

\section{Introduction}

Upper tract urothelial carcinoma (UTUC) is a relatively rare disease, and accounts for approximately $5-10 \%$ of all urothelial cancers (UC). In western countries the incidence is low, with approximately $1-2$ cases per 100,000 and mainly diagnosed in older male patients $(1,2)$. In some Asian countries, the proportion of UTUC is reported in up to $25 \%$ of all UC patients and the majority diagnosed at an invasive stage (3).

Preoperative staging of UTUC is difficult, due to challenges in obtaining adequate tissue and suboptimal imaging $(4,5)$, which can lead to difficulties in selecting patients who might benefit from neoadjuvant chemotherapy (NAC). Pathologic evaluation of small ureterorenoscopic biopsies can be demanding. Tumor grade can be determined correctly in the majority of patients with high grade UTUC, with a concordance between endoscopic biopsy 
and nephroureterectomy specimen of up to $>90 \%(6,7)$; upgrading of low grade biopsies occurs in a significant number of patients (7-9). Determination of tumor stage is accepted to be unreliable based on biopsy only $(6,8)$. Therefore, UTUC is stratified based on risk factors, which are known to worsen prognosis. Surgical treatment recommendation, especially in terms of radicality of surgery and need for lymphadenectomy, is based on these risk categories (10-13).

The EAU guidelines refer to low risk tumors in case of unifocal disease, tumor size $<2 \mathrm{~cm}$, low grade cytology and biopsy, and no invasive aspects on computerized urography. All other tumors are considered as high-risk disease, especially if hydronephrosis or variant histology are present, as well as in patients who previously underwent radical cystectomy (RC) for high-grade cancer $(10,14)$. Radical nephroureterectomy (RNU) is the standard for patients with high-risk disease. Though, one out of five patients treated with RNU for UTUC will experience disease recurrence, of which the majority of patients ( $82 \%$ ) will die of the disease $(15,16)$.

In UC, cisplatin-based NAC is standard for muscleinvasive urothelial bladder cancer (UBC) $(17,18)$, but no evidence from randomized phase 3 trials is available for UTUC. In contrast, level 1 evidence for adjuvant chemotherapy (AC) is still lacking in high-risk UBC $(18,19)$. Just recently, the POUT trial reported a significantly improved event-free survival (EFS) for patients undergoing platinum-based AC after RNU for UTUC (20).

In metastatic urothelial cancer (mUC), the standard first line treatment for eligible patients, who did not undergo $\mathrm{NAC}$ or AC is a cisplatin-based combination chemotherapy; recommendations for ineligible patients include either a carboplatin-based chemotherapeutic regimen or treatment with the immune-checkpoint inhibitors (CPI) atezolizumab or pembrolizumab, depending on the PD-L1 status of the tumor (21).

Rationale for this review is to give a summary of the current systemic treatment options for urothelial cancer and specific considerations for UTUC. Data for patients with metastatic UTUC is sparse and treatment recommendations are mostly based on extrapolation from $\mathrm{mUC}$ or on subgroups of patients with UTUC from clinical trials or retrospective cohort analysis (10). We present the following article in accordance with the Narrative Review reporting checklist (available at https:// dx.doi.org/10.21037/tau-21-47).

\section{Methods}

To identify relevant published data, we used the PubMed/ Medline search engine to conduct an online literature search using the terms (systemic therapy OR chemotherapy OR check-point inhibition OR targeted therapy OR trial) AND (urothelial cancer OR upper tract urothelial carcinoma) AND (management OR therapy OR treatment).

Only articles in English language were retrieved. The authors reviewed the extant articles with the intention to include the relevant data in this review. A manual search from authors' bibliographies was also used, when felt to be expedient. In order to identify ongoing neoadjuvant and adjuvant clinical trials of interest that have not been published yet, we accessed the clinicaltrials.gov online databank and provide the NCT number when appropriate. The search term 'upper tract urothelial carcinoma OR urothelial cancer' was used. Studies retrieved were screened and included if the study patients were diagnosed UTUC.

\section{Discussion}

\section{Perioperative systemic therapy}

\section{NAC}

In muscle-invasive bladder cancer (MIBC), the use of cisplatin-based NAC is supported by level 1 evidence, showing a statistically significant and clinically meaningful survival benefit (18). In contrast, there is only limited data available for high-risk UTUC from smaller retrospective and prospective series. Randomized phase 3 trials are ongoing, though patient recruitment can be challenging, in part, because of the rarity of the disease (22-24).

Retrospective analysis of a large cohort of patients who underwent RNU $(n=1,363)$ revealed that use of NAC for high-risk UTUC is low, with only $3.4 \%$ of patients undergoing chemotherapy before radical surgery (3).

Though, the rational to use NAC for treatment of nonmetastatic high-risk UTUC might be even greater than that in MIBC, as only half of patients undergoing RNU are eligible for a cisplatin-based chemotherapy before surgery and only one out of five patients remains eligible after RNU (25), mostly due to deterioration of renal function (26). In addition, NAC allows evaluation of pathological downstaging as prognostic marker (27). While several clinical trials are on the way (28), level 1 evidence for NAC in UTUC is still lacking (Table 1).

However, there are also multiple concerns regarding the 
Table 1 Neoadjuvant trials for UTUC

\begin{tabular}{|c|c|c|c|c|c|}
\hline Treatment & Identifier & Cohort & Clinical phase & Sample size & Primary endpoint \\
\hline Gem/Cis & NCT02876861 & High grade & II & 50 & OS \\
\hline Gem/Cis & NCT04574960 & High grade & II & 14 & Safety \\
\hline Gem/Cis & NCT01261728 & High grade & ॥ & 54 & $\mathrm{pCR}$ \\
\hline Gem/Carbo or ddMVAC & NCT02412670 & High grade & II & 60 & $\mathrm{pCR}$ \\
\hline \multicolumn{6}{|l|}{ Combination therapy } \\
\hline Durvalumab + Tremelimumab & NCT02812420 & High risk, cisplatin-ineligible & II & 15 & Safety \\
\hline Durvalumab + Gem/Platin & NCT04617756 & High risk & $\|$ & 99 & $\mathrm{pCR}$ \\
\hline \multicolumn{6}{|l|}{ Other } \\
\hline Tislelizumab & NCT04672330 & High grade & II & 20 & $\mathrm{pCR}$ \\
\hline Infigratinib & NCT04228042 & Low and high grade, platin-ineligible & $\mathrm{lb}$ & 20 & Safety \\
\hline
\end{tabular}

UTUC, upper tract urothelial carcinoma; Gem/Cis, Gemcitabine/Cisplatin; Carbo, Carboplatin; MVAC: methotrexate, vinblastine, doxorubicin, cisplatin; ddMVAC, dose dense MVAC; OS, overall survival; pCR, pathologic complete response; DFS, disease free survival.

use of NAC in patients diagnosed with UTUC. Beside a potential increased peri-operative morbidity, there is a risk of a delay to definitive surgery, especially in patients not responding to NAC. One of the major clinical challenges is the quality of the primary biopsy and imaging methods used, which might result in difficulties to reliably identify invasive disease, leading to potential over-treatment.

Matin et al. evaluated the incidence of pathologic downstaging and pathological complete response (pCR) in patients with high-grade UTUC. Significant downstaging to the level of pCR was described in $14 \%$ of patients after NAC (29).

A multi-institutional, prospective, two-armed phase 2 trial investigated pathological complete response (pCR) after NAC for high grade UTUC. While the gemcitabine/carboplatin arm closed early due too poor accrual, the majority of patients included in the accelerated methotrexate, vinblastine, doxorubicin, and cisplatin (MVAC) arm have completed all planned treatment cycles and achieved a pCR in $13.8 \%$ (90\% CI: $4.9-28.8 \%)$ of patients (27).

Recent systematic reviews and meta-analyses found a pCR rate (ypT0N0M0) of $11 \%$ (95\% CI: $8-15 \%)$, a pathologic partial response rate (pPR; $\leq$ ypT1N0M0) of
$43 \%$ (95\% CI: 34-52\%) and a pathological downstaging (c'T $>\mathrm{pT}$ ) in $33 \%$ (95\% CI: $14-52 \%)$ of cases (30). NAC was associated with higher rates of pathological downstaging (RR 6.48, 95\% CI: 2.05-20.44, $\mathrm{P}=0.001)$ and $\mathrm{pCR}(\mathrm{RR}$ 18.46, 95\% CI: 3.34-99.24, $\mathrm{P}=0.001$ ) (31) resulting in an improvement in OS of $56 \%$ (HR 0.44; 95\% CI: 0.32-0.59) and CSS of $62 \%$ (HR 0.38 ; 95\% CI: 0.24-0.61) (30). In addition, pathological downstaging was found to be the strongest prognostic factor for recurrence-free survival (RFS; HR 0.2, $\mathrm{P}<0.001$ ), cancer specific survival (CSS; HR 0.19, $\mathrm{P}<0.001$ ) and overall survival (OS; HR 0.40, $\mathrm{P}<0.001)$ in an international observational study in patients undergoing NAC and RNU for high risk UTUC (32).

Several ongoing clinical trials are currently evaluating NAC in UTUC (Table 1) including the multi-centric URANUS trial, which aims to explore the feasibility of perioperative chemotherapy in UTUC at various European centers (NCT02969083). Patients who are eligible for a cisplatin-based chemotherapy are randomized to receive either cisplatin-based NAC or AC. Patients ineligible for cisplatin-based perioperative chemotherapy undergo RNU only.

Despite the lack of level 1 evidence, the cumulative evidence suggests that NAC for locally advanced UTUC 
can improve oncological outcome. Nevertheless, prospective studies are needed to clarify the clinical benefit of NAC for patients with locally advanced UTUC (33).

\section{Neoadjuvant immunotherapy}

In the neoadjuvant setting of UBC, two single-arm, phase 2 trials with pembrolizumab (PURE-01) (34) and atezolizumab (ABACUS) (35) in patients with nonmetastatic or locally advanced UBC have been published recently. These trials included unselected patients and used a comparatively low number of treatment cycles in order to not delay potentially curative surgery (34-37). Though, no patients with UTUC were included into these trials.

At time of RC, neoadjuvant CPI treatment resulted in a pCR rate of $31 \%$ and $42 \%$ for atezolizumab and pembrolizumab, respectively. Response rates were dependent on tumor PD-L1 expression and increased in patients with $\mathrm{PD}-\mathrm{L} 1$ positive tumors compared to those with PD-L1 negative tumors [37\% vs. $25 \%$ for ABACUS (35) and $54 \%$ vs. $13 \%$ for PURE-01 (34)]. Whether these improved pCR rates to neoadjuvant CPI according to PD-L1 status translate into a survival benefit, has to be determined. The adverse events were similar to those observed in clinical trials evaluating CPI in metastatic UC and no delay, nor failure to undergo $\mathrm{RC}$ was noted in both trials $(34,35)$.

With PURE-02, a multi-center prospective phase 2, single-arm trial is ongoing to evaluate pembrolizumab in the neoadjuvant setting in UTUC (38). This trial could have the potential to increase the generally low acceptance of neoadjuvant therapy in cancers of the upper and lower urothelial tract (39). In addition, several ongoing clinical trials evaluate the neoadjuvant treatment with novel targeting agents as monotherapy or in combination with chemotherapy (Table 1).

\section{Adjuvant chemotherapy}

Decision making about administration of $\mathrm{AC}$ after RNU is based on the information from the definitive pathological specimen with the aim to avoid over-treatment. However, RNU before systemic therapy may result in the exclusion of a significant number of patients. Analysis of the National Cancer Data Base (NCDB) between 2004 and 2012 revealed that only $23.54 \%$ of patients with pT3/4 and/or pN+ UTUC who underwent RNU received AC (40). Just recently, a randomized phase 3 clinical trial (POUT) has been published, demonstrating a significantly improved EFS in patients with locally advanced UTUC, when being treated with adjuvant gemcitabine/platin-based chemotherapy (20).

The trial was closed early after recruitment of 261 patients, since the pre-planned interim analysis met the criterion for early closure. With a median follow-up of 30.3 months (IQR, 18.0-47.5 months), the 3-year EFS estimates were $71 \%$ (95\% CI: 61-78\%) and 46\% (95\% CI: $36-56 \%)$ for patients receiving AC or surveillance, respectively. AC resulted in a $55 \%$ reduction in relative risk of disease recurrence or death (HR 0.45, 95\% CI: 0.30-0.68). Median disease-free survival among patients allocated to surveillance was 29.8 months (IQR, 6.3-not reached; 95\% CI: 13.6-incalculable), and was not reached among patients undergoing AC. The trial design allowed dose-reductions or switch to a carboplatin-based regimen in patients, initially planned for cisplatin-based combination chemotherapy (20).

The majority of the 126 patients who started chemotherapy completed four cycles of AC (75\%); there was no difference between the two pre-planned chemotherapy regimens. Thirty-one participants discontinued chemotherapy before receiving the planned four cycles and were delivered without any dose reduction in $91 \%(198 / 218)$ of the gemcitabine/cisplatin patients and $83 \%(186 / 223)$ of the gemcitabine/carboplatin patients. Sixteen patients $(21 \%)$ intended to receive cisplatin-based AC had to be switched to carboplatin because of a drop in GFR after randomization (20).

The relative effect on survival of carboplatin- and cisplatin-based chemotherapies remains unclear in $\mathrm{UC}$, since there is no sufficient data from randomized clinical trials comparing the two agents. The POUT trial underlines the benefit of adjuvant platinum-based chemotherapy for patients who underwent RNU for locally advanced or lymphogenic metastasized UTUC. While cisplatin should be the preferred agent for an AC; patients ineligible for cisplatin might still benefit from a carboplatinbased regimen (20).

In addition to this level 1 evidence, a recently published systematic review and meta-analysis (30) including 29 studies with two prospective randomized clinical trials $(20,41)$, found that AC resulted in a $23 \%$ OS benefit (HR 0.77; 95\% CI: 0.64-0.92), a 21\% CSS benefit (HR 0.79; 95\% CI: $0.69-0.91$ ) and a 48\% (HR 0.52; 95\% CI: 0.38 0.70 ) improvement in disease-free survival (DFS) (30).

In a recent meta-analysis, $\mathrm{AC}$ was associated with improved metastasis-free survival (HR 0.65, 95\% CI: 0.55-0.76, P<0.001) and CSS (HR 0.66, 95\% CI: 0.57-0.77, 
Table 2 Adjuvant trials for UTUC

\begin{tabular}{|c|c|c|c|c|c|}
\hline Treatment & Identifier & Cohort & Clinical phase & Sample size & Primary endpoint \\
\hline Gem/Cis or MVAC & NCT02969083 & High risk & II & 200 & DFS \\
\hline Gem/Cis or Gem/Carbo & NCT01993979 & pT2-4 pN0-3 M0; pTany N1-3 M0 & III & 261 & DFS \\
\hline \multicolumn{6}{|l|}{ Targeted therapies } \\
\hline
\end{tabular}

UTUC, upper tract urothelial carcinoma; Gem/Cis, Gemcitabine/Cisplatin; Carbo, Carboplatin; MVAC: methotrexate, vinblastine, doxorubicin, cisplatin; DFS, disease free survival.

$\mathrm{P}<0.001$ ), also in patients with locally advanced UTUC; indeed, the association of AC with OS was statistically significant only in patients with locally advanced UTUC (31).

\section{Adjuvant immunotherapy}

With IMvigor010, CheckMate274 and AMBASSADOR, there are three major randomized phase 3 clinical trials evaluating the CPIs atezolizumab, nivolumab or pembrolizumab in patients with high-risk UBC or UTUC. IMvigor010 has failed to show an improvement in RFS (42), while an exploratory biomarker analysis showed that post-surgical circulating tumor DNA (ctDNA) was found in $37 \%$ of the included patients. The interim OS analysis favored atezolizumab in ctDNA-positive patients (stratified HR 0.58; 95\% CI: 0.39-0.85; $\mathrm{P}=0.005$ ) with a median OS of 25.8 months for patients treated with atezolizumab compared to 15.8 months for patients on observation only (43).

Nivolumab has been evaluated in the adjuvant setting of high-risk urothelial cancer in the randomized, placebocontrolled CheckMate274 trial. Patients were eligible with presence of muscle-invasive disease after NAC followed by radical surgery or locally advanced disease or local lymph node metastasis. In addition, patients unwilling to undergo or ineligible for adjuvant cisplatin-based chemotherapy were included (44). This trial also included 149 patients with UTUC (21\%). Subgroup analysis reveals a clear benefit in RFS for patients with UBC [HR 0.62 (0.49-0.78)]. In patients with UTUC, the effect is not as distinct and independent of the tumor location. Both groups of patients, with either tumor in the renal pelvis $(n=96)$ or the ureter $(\mathrm{n}=53)$, did not show a benefit in RFS when receiving nivolumab $v s$. placebo (44), which raises the question, if patients with UTUC unfit for cisplatin-based AC should undergo adjuvant treatment with nivolumab, or not.
In addition, several clinical trials in the adjuvant therapeutic setting are ongoing to evaluate different chemotherapeutic regimens or novel targeting agents (Table 2).

\section{Systemic therapy of metastatic disease}

Over the last years, significant progress in systemic therapy has been made with the introduction of CPI and targeted therapies into the treatment landscape. Though, cisplatinbased combination chemotherapy remains the standard first line therapy for patients with metastatic UC, who are eligible for cisplatin combined with either gemcitabine (GC) or MVAC.

Due to the rarity of the disease, only very limited data from clinical trials evaluating systemic therapies in metastatic UTUC are available. Recommendations are mostly based on extrapolation of data from UBC or small subgroups of patients with UTUC included in these clinical trials.

Importantly, the majority of patients with metastatic UTUC suffering renal impairment due to hydronephrosis, infiltration of the renal parenchyma depending on tumor location, or previous radical surgery.

\section{Chemotherapy}

Platinum-based combination chemotherapy regimens are the standard for eligible patients with $\mathrm{mUC}$ in the first line setting. Trials including patients with newly diagnosed mUC have shown a median OS of approximately 15 and 10 months for patients receiving cisplatin- and carboplatinbased chemotherapeutics, respectively $(45,46)$. These results have been confirmed and even exceeded in terms of ORR for carboplatin-based regimens in chemotherapy-only arms from recent clinical trials [i.e., in IMvigor130 (47), KEYNOTE-361 (48), and DANUBE (49)]. These trials are comparing CPI single agents, CPI-chemo or CPI-CPI 
combinations to first line platinum-based chemotherapy. Data on treatment of metastatic UTUC is sparse and recommendations are mainly based on experience, extrapolated from metastatic UBC (10).

Recently, an individual patient data meta-analysis supported this approach, as location of the primary tumor within the urothelial tract was not associated with differential response to platin-based chemotherapy (50).

In addition, patients with $\mathrm{mUC}$, who have not progressed on first line platin-based chemotherapy, had a clinically meaningful improved OS, when chemotherapy was followed by treatment with avelumab. This switch to maintenance therapy led to a median OS of $21.4 v s$. 14.3 months in those who did not receive maintenance therapy (HR 0.69; 95\% CI: 0.56-0.86) (51). The trial included 187 patients with UTUC (27\%), while no specific data on this subgroup has been published so far.

Second line chemotherapy for mUC with vinflunine shows a marginal survival benefit over best supportive care; it has been approved by the European Medical Agency (EMA) for the post-platinum setting, meanwhile being relegated to the third line, post-CPI setting (52).

\section{Immunotherapy}

CPI have broadened the therapeutic options of $\mathrm{mUC}$ and have been approved as first line treatment in cisplatin-ineligible patients with $\mathrm{PD}-\mathrm{L} 1$ positive tumors (atezolizumab, pembrolizumab) by both FDA and EMA $(53,54)$; and in patients ineligible for chemotherapy (FDA) independent of PD-L1 expression.

In addition, five CPIs (avelumab, durvalumab, pembrolizumab, atezolizumab, and nivolumab) have been approved for the treatment of mUC after platinum-based chemotherapy by the FDA, of which three (pembrolizumab, atezolizumab, and nivolumab) have also been approved by the EMA. Pembrolizumab is the only CPI with level 1 evidence in the second line, post-platinum setting, derived from a prospective randomized phase 3 clinical trial (KEYNOTE-045) (55). Atezolizumab failed to reach the primary endpoint in IMvigor211 (56) and all other CPIs have been granted initial accelerated approval based on single-arm, phase 2 trials (57-59).

All of these trials included both, patients with UBC and UTUC, and improved objective response rates (ORR) for patients with UTUC have been reported in some of the trials.
In first line setting, IMvigor210 (cohort 1) included 33 patients with UTUC (27.7\% of the cohort). In this subgroup, a favorable ORR of $39 \%$ (95\% CI: $17-51 \%$ ) compared to patients with lower urinary tract tumors (LTUC), for who the ORR was 17\% (95\% CI: 9-26\%) (53). Though, data is not consistent with KEYNOTE-052, the second single-arm, phase 2 trial, that lead to accelerated approval of pembrolizumab. In this trial, 19\% of the entire cohort (69/370) had an UTUC. The ORR was 22\% (95\% CI: $12-35 \%)$ for those patients compared to $28 \%$ (95\% CI: $23-34 \%$ ) in patients with LTUC (54).

The phase 3 clinical trial KEYNOTE-361, evaluated the pembrolizumab in the first line setting either as monotherapy or in combination with platin-based combination chemotherapy and compared it to combination chemotherapy alone. The trial did not reach its prespecified level of significance for the primary endpoint. The subgroup of patients with UTUC (21\%; 146/703 patients) shows a significant improvement in PFS when treated with the combination over chemotherapy alone, though this does not translate into a benefit in OS (48).

IMvigor130, which evaluated atezolizumab in a similar setting in a phase 3 clinical trial, included patients with mUC, of who $26 \%(312 / 1,213$ patients) harbored an UTUC (26\%). The trial showed a statistically significant improvement in PFS, though it failed to show a significant improvement in OS for both the combination of atezolizumab with platin-based chemotherapy or atezolizumab alone compared tox first line platin-based combination. Data in the subgroup of patients with UTUC have not been published so far (47).

The DANUBE phase 3 trial evaluated durvalumab alone or in combination with tremelimumab versus platinumbased chemotherapy in the first line setting of mUC including 221 patients ( $21 \%$ of patients) with UTUC. The trial did not reach either of the co-primary endpoints of overall survival and did not report separate results for the subgroup of patients with UTUC (49).

In the second line setting, subgroup analysis of KEYNOTE-045 revealed a trend towards an improved OS to pembrolizumab compared to second line chemotherapy in patients with UTUC (HR 0.53; 95\% CI: 0.28-1.01), which was even more pronounced than that in LTUC patients (HR 0.77; 95\% CI: 0.60-0.97) (55). Conversely, this effect was not observed in the IMvigor211 trial, which compared atezolizumab to second line chemotherapy (56). 


\section{Novel targeted therapies}

Due to the commonly found renal impairment in patients with metastatic UTUC, novel targeted therapeutical approaches, which are not dependent on the renal function, are of special interest. Genetic characterization revealed that UTUC is often associated with luminal-papillary characteristics and harbors high FGFR3, which correlates with a T-cell depleted immune microenvironment (60) and therefore offers the potential as target for novel therapies.

Recently, an increasing number of clinical trials are evaluating targeting agents (61). The pan-FGFR targeting erdafitinib and the novel antibody-drug conjugate (ADC) enfortumab vedotin (EV) have both been granted accelerated approval for $\mathrm{mUC}$ by the FDA.

Results from BLC2001, a single-arm, phase 2 trial evaluating the tyrosine kinase inhibitor of FGFR1-4 erdafitinib has recently been published. Patients with locally advanced or metastatic UC who harbor a FGFR3 mutation or FGFR2/3 fusion were included; either treatment naïve patients, who were ineligible for cisplatin-based combination chemotherapy or those who experienced disease progression to at least one previous chemotherapeutic were included. Treatment with erdafitinib resulted in an ORR of $40 \%$ with a median PFS and OS of 5.5 and 13.8 months, respectively. The response rate in patients $(\mathrm{n}=22)$ previously treated with CPI was even higher (59\%) (62).

$\mathrm{EV}$ is an ADC targeting the surface protein Nectin-4, which is highly expressed in UC. Recently, results from several early clinical trials have been published with promising results and phase 3 trials are ongoing in mUC. In the EV-101 trial, patients with solid tumors expressing Nectin-4, including 155 patients with heavily pretreated mUC, treatment with EV showed clinically meaningful and durable response to $\mathrm{EV}$. An ORR of $43 \%$ resulted in a median duration of response of 7.4 months, a median OS of 12.3 months, and a 12 -month OS rate of $51.8 \%$ (63).

$\mathrm{EV}$ has also been investigated in a phase 2 clinical trial (EV201), including patients with locally advanced or metastatic UC, who have been pre-treated with CPI and platinumbased chemotherapy (cohort 1). With an ORR of $42 \%$, including $9 \%$ of patients showing CR, the OS rates were $50.4 \%$ and $34.2 \%$ at 12 and 18 months, respectively (64). Based on these results, combined with a manageable safety profile, EV was granted accelerated FDA approval for this indication.

These results have been recently confirmed in the phase 3 trial of EV for the treatment of patients with locally advanced or metastatic urothelial carcinoma who had previously received platinum-containing chemotherapy and had disease progression during or after treatment with a PD-1 or PD-L1 inhibitor (EV-301).

Progression-free $[5.55$ vs. 3.71 months; HR (95\% CI), 0.62 (0.51 to 0.75$) ; \mathrm{P}<0.001]$ and overall survival $[12.88$ vs. 8.97 months; HR (95\% CI) 0.70 (0.56 to 0.89 ); $\mathrm{P}=0.001$ ] were longer in the $\mathrm{EV}$ group than in the chemotherapy group. The clinical trial included 205 patients with UTUC (34\%). Subgroup analysis based on location of the primary tumor revealed a significant benefit in terms of OS in patients with UBC [HR $0.67(0.51-0.88)]$, while in patients with UTUC it did not reach statistical significance [HR 0.85 (0.57-1.27)] (65).

In first line treatment of $\mathrm{mUC}$, the combination of $\mathrm{EV}$ with pembrolizumab in cisplatin- ineligible patients is under evaluation, with preliminary results from this phase $1 \mathrm{~b}$ trial (EV-103). Indeed, the combination showed an ORR of $73.3 \%$, with $15.6 \%$ CR (66); a phase 3 trial with this combination therapy is ongoing (NCT04223856, EV-302).

\section{Future directions and conclusions}

Recent genomic and gene expression studies in localized and metastatic UTUC, revealed a number of altered molecular pathways, such as FGFR3, TP53, or germline mutations in mismatch repair (MMR) genes that might have implications for future targeted therapies in the perioperative (Tables 1,2) and the metastatic treatment setting $(67,68)$. Further research is needed of this molecular and genomic profiling of UTUC can be implemented in the treatment algorithm thereby leading to improved survival outcomes in patients with this devastating disease.

Over the next years, results of several phase 2 and 3 clinical trials are expected to provide additional treatment options for metastatic UC including patients with UTUC. These trials evaluate front-line CPI compared to chemotherapy, alone or in combination, FGFR targeting agents and antibody-drug conjugates. Furthermore, extensive research of predictive biomarkers is ongoing to guide a more individualized treatment approach for each patient. Several biomarkers such as microsatellite instability (MSI), deficiencies in DNA damage repair genes (DDR), and tumor mutational burden (TMB) are under investigation to predict response to those therapies, and targetable genetic alterations, such as EGFR, ERBB2, ERBB3, PIK3CA, and RAS have been found as potential 
oncogenic drivers in subsets of UC (69), with the potential as novel therapeutics.

Clinical trials evaluating the use of neoadjuvant immune checkpoint inhibitors in patients with high risk UTUC are also on their way with the potential to increase the generally low acceptance of neoadjuvant therapy in UTUC. New therapeutic options and the potential implementation of biomarkers to guide patient selection are likely to reshape the treatment landscape for UC in the near future. Special attention to the differential impact of these therapies on UTUC versus LTUC will be required to help build solid evidence for evidence-based systemic treatment of UTUC.

\section{Acknowledgments}

Funding: None.

\section{Footnote}

Provenance and Peer Review: This article was commissioned by the Guest Editor (Tilman Todenhöfer) for the series "Management of Advanced Genitourinary Malignancies" published in Translational Andrology and Urology. The article has undergone external peer review.

Reporting Checklist: The authors have completed the Narrative Review reporting checklist. Available at https:// dx.doi.org/10.21037/tau-21-47

Peer Review File: Available at https://dx.doi.org/10.21037/ tau-21-47

Conflicts of Interest: All authors have completed the ICMJE uniform disclosure form (available at https://dx.doi. org/10.21037/tau-21-47). The series "Management of Advanced Genitourinary Malignancies" was commissioned by the editorial office without any funding or sponsorship. Dr. KMG reports receiving payment or honoraria from Astellas, Astra Zeneca, BMS, Ipsen, Janssen, Merck, MSD and Roche; support for attending meetings and/or travel from Allergan, Astellas, Astra Zeneca, Bayer, BMS, Janssen, Merck, MSD, Novartis, Pfizer, Pierre Fabre and Roche; Dr. KMG is on the Advisory Board of BMS, Ferring, MSD, Janssen and Roche and is the Head of Austrian UroOncology Group (AUO). Prof. SFS reports receiving both consulting fees and honoraria from Astellas, Astra Zeneca, Bayer, BMS, Cepheid, Ferring, Ipsen, Janssen, Lilly, MSD, Olympus, Pfizer, Pierre Fabre, Richard Wolf, Roche,
Sanochemia, Sanofi, Takeda and Urogen; Prof. SFS reports to be on the speakers' bureau of Astellas, Astra Zeneca, Bayer, BMS, Cepheid, Ferring, Ipsen, Janssen, Lilly, MSD, Olympus, Pfizer, Pierre Fabre, Richard Wolf, Roche, Sanochemia, Sanofi, Takeda and Urogen; Prof. SFS has a patent "Soluble Fas urinary marker for the detection of bladder". The authors have no other conflicts of interest to declare.

Ethical Statement: the authors are accountable for all aspects of the work in ensuring that questions related to the accuracy or integrity of any part of the work are appropriately investigated and resolved.

Open Access Statement: This is an Open Access article distributed in accordance with the Creative Commons Attribution-NonCommercial-NoDerivs 4.0 International License (CC BY-NC-ND 4.0), which permits the noncommercial replication and distribution of the article with the strict proviso that no changes or edits are made and the original work is properly cited (including links to both the formal publication through the relevant DOI and the license). See: https://creativecommons.org/licenses/by-nc-nd/4.0/.

\section{References}

1. Siegel RL, Miller KD, Jemal A. Cancer statistics, 2020. CA Cancer J Clin 2020;70:7-30.

2. Soria F, Shariat SF, Lerner SP, et al. Epidemiology, diagnosis, preoperative evaluation and prognostic assessment of upper-tract urothelial carcinoma (UTUC). World J Urol 2017;35:379-87.

3. Margulis V, Shariat SF, Matin SF, et al. Outcomes of radical nephroureterectomy: a series from the Upper Tract Urothelial Carcinoma Collaboration. Cancer 2009;115:1224-33.

4. Favaretto RL, Shariat SF, Savage C, et al. Combining imaging and ureteroscopy variables in a preoperative multivariable model for prediction of muscle-invasive and non-organ confined disease in patients with upper tract urothelial carcinoma. BJU Int 2012;109:77-82.

5. Janisch F, Shariat SF, Baltzer P, et al. Diagnostic performance of multidetector computed tomographic (MDCTU) in upper tract urothelial carcinoma (UTUC): a systematic review and meta-analysis. World J Urol 2020;38:1165-75.

6. Rojas CP, Castle SM, Llanos CA, et al. Low biopsy volume in ureteroscopy does not affect tumor biopsy 
grading in upper tract urothelial carcinoma. Urol Oncol 2013;31:1696-700.

7. Freund JE, Duivenvoorden MJC, Sikma BT, et al. The Diagnostic Yield and Concordance of Ureterorenoscopic Biopsies for Grading of Upper Tract Urothelial Carcinoma: A Dutch Nationwide Analysis. J Endourol 2020;34:907-13.

8. Smith AK, Stephenson AJ, Lane BR, et al. Inadequacy of biopsy for diagnosis of upper tract urothelial carcinoma: implications for conservative management. Urology 2011;78:82-6.

9. Dev HS, Poo S, Armitage J, et al. Investigating upper urinary tract urothelial carcinomas: a single-centre 10-year experience. World J Urol 2017;35:131-8.

10. Rouprêt M, Babjuk M, Burger M, et al. European Association of Urology Guidelines on Upper Urinary Tract Urothelial Carcinoma: 2020 Update. Eur Urol 2021;79:62-79.

11. Chromecki TF, Cha EK, Fajkovic H, et al. The impact of tumor multifocality on outcomes in patients treated with radical nephroureterectomy. Eur Urol 2012;61:245-53.

12. Favaretto RL, Shariat SF, Chade DC, et al. The effect of tumor location on prognosis in patients treated with radical nephroureterectomy at Memorial Sloan-Kettering Cancer Center. Eur Urol 2010;58:574-80.

13. Remzi M, Haitel A, Margulis V, et al. Tumour architecture is an independent predictor of outcomes after nephroureterectomy: a multi-institutional analysis of 1363 patients. BJU Int 2009;103:307-11.

14. Xylinas E, Rink M, Robinson BD, et al. Impact of histological variants on oncological outcomes of patients with urothelial carcinoma of the bladder treated with radical cystectomy. Eur J Cancer 2013;49:1889-97.

15. Rink M, Sjoberg D, Comploj E, et al. Risk of cancerspecific mortality following recurrence after radical nephroureterectomy. Ann Surg Oncol 2012;19:4337-44.

16. Rouprêt M, Hupertan V, Seisen T, et al. Prediction of cancer specific survival after radical nephroureterectomy for upper tract urothelial carcinoma: development of an optimized postoperative nomogram using decision curve analysis. J Urol 2013;189:1662-9.

17. Yin M, Joshi M, Meijer RP, et al. Neoadjuvant Chemotherapy for Muscle-Invasive Bladder Cancer: A Systematic Review and Two-Step Meta-Analysis. Oncologist 2016;21:708-15.

18. Witjes JA, Bruins HM, Cathomas R, et al. European Association of Urology Guidelines on Muscle-invasive and Metastatic Bladder Cancer: Summary of the 2020
Guidelines. Eur Urol 2021;79:82-104.

19. Sternberg CN, Skoneczna I, Kerst JM, et al. Immediate versus deferred chemotherapy after radical cystectomy in patients with pT3-pT4 or N+ M0 urothelial carcinoma of the bladder (EORTC 30994): an intergroup, open-label, randomised phase 3 trial. Lancet Oncol 2015;16:76-86.

20. Birtle A, Johnson M, Chester J, et al. Adjuvant chemotherapy in upper tract urothelial carcinoma (the POUT trial): a phase 3, open-label, randomised controlled trial. Lancet 2020;395:1268-77.

21. Gust KM, Abufaraj M, D'Andrea D, et al. Second line immune checkpoint inhibition in urothelial cancer. Transl Androl Urol 2019;8:414-20.

22. Hosogoe S, Hatakeyama S, Kusaka A, et al. Platinumbased Neoadjuvant Chemotherapy Improves Oncological Outcomes in Patients with Locally Advanced Upper Tract Urothelial Carcinoma. Eur Urol Focus 2018;4:946-53.

23. Kubota Y, Hatakeyama S, Tanaka T, et al. Oncological outcomes of neoadjuvant chemotherapy in patients with locally advanced upper tract urothelial carcinoma: a multicenter study. Oncotarget 2017;8:101500-8.

24. Porten S, Siefker-Radtke AO, Xiao L, et al. Neoadjuvant chemotherapy improves survival of patients with upper tract urothelial carcinoma. Cancer 2014;120:1794-9.

25. Kaag MG, O'Malley RL, O'Malley P, et al. Changes in renal function following nephroureterectomy may affect the use of perioperative chemotherapy. Eur Urol 2010;58:581-7.

26. Kaag M, Trost L, Thompson RH, et al. Preoperative predictors of renal function decline after radical nephroureterectomy for upper tract urothelial carcinoma. BJU Int 2014;114:674-9.

27. Margulis V, Puligandla M, Trabulsi EJ, et al. Phase II Trial of Neoadjuvant Systemic Chemotherapy Followed by Extirpative Surgery in Patients with High Grade Upper Tract Urothelial Carcinoma. J Urol 2020;203:690-8.

28. Azizi M, Cheriyan SK, Peyton CC, et al. Optimal Management of Upper Tract Urothelial Carcinoma: an Unmet Need. Curr Treat Options Oncol 2019;20:40.

29. Matin SF, Margulis V, Kamat A, et al. Incidence of downstaging and complete remission after neoadjuvant chemotherapy for high-risk upper tract transitional cell carcinoma. Cancer 2010;116:3127-34.

30. Leow JJ, Chong YL, Chang SL, et al. Neoadjuvant and Adjuvant Chemotherapy for Upper Tract Urothelial Carcinoma: A 2020 Systematic Review and Meta-analysis, and Future Perspectives on Systemic Therapy. Eur Urol 2021;79:635-54. 
31. Quhal F, Mori K, Sari Motlagh R, et al. Efficacy of neoadjuvant and adjuvant chemotherapy for localized and locally advanced upper tract urothelial carcinoma: a systematic review and meta-analysis. Int J Clin Oncol 2020;25:1037-54.

32. Foerster B, Abufaraj M, Petros F, et al. Efficacy of Preoperative Chemotherapy for High Risk Upper Tract Urothelial Carcinoma. J Urol 2020;203:1101-8.

33. Gust KM, Shariat SF. Re: High Response Rates to Neoadjuvant Chemotherapy in High-Grade Upper Tract Urothelial Carcinoma. PractiseUpdate (accessed online May 31st 2019). 2019.

34. Necchi A, Anichini A, Raggi D, et al. Pembrolizumab as Neoadjuvant Therapy Before Radical Cystectomy in Patients With Muscle-Invasive Urothelial Bladder Carcinoma (PURE-01): An Open-Label, Single-Arm, Phase II Study. J Clin Oncol 2018;36:3353-60.

35. Powles T, Kockx M, Rodriguez-Vida A, et al. Clinical efficacy and biomarker analysis of neoadjuvant atezolizumab in operable urothelial carcinoma in the ABACUS trial. Nat Med 2019;25:1706-14.

36. Powles T, Rodriguez-Vida A, Duran I, et al. A phase II study investigating the safety and efficacy of neoadjuvant atezolizumab in muscle invasive bladder cancer (ABACUS). J Clin Oncol 2018;36:abstr 4506.

37. Necchi A, Briganti A, Raggi D, et al. Interim results from PURE-01: A phase 2, open-label study of neoadjuvant pembrolizumab (pembro) before radical cystectomy for muscle-invasive urothelial bladder carcinoma (MIUC). J Clin Oncol 2018;36:TPS533.

38. Necchi A, Raggi D, Gust K, et al. PURE-02: An open label, multicenter, single-arm, phase 2 study of neoadjuvant pembrolizumab (Pembro), preceding radical nephroureterectomy (RNU), for patients with localized high-risk urothelial carcinoma of the upper urinary tract (UTUC). European Urology Supplements 2019;18:e1835.

39. Gust KM, Shariat SF. Re: Pembrolizumab as Neoadjuvant Therapy Before Radical Cystectomy in Patients with Muscle-invasive Urothelial Bladder Carcinoma (PURE01): An Open-label, Single-arm, Phase II Study. Eur Urol 2019;75:695-6.

40. Seisen T, Krasnow RE, Bellmunt J, et al. Effectiveness of Adjuvant Chemotherapy After Radical Nephroureterectomy for Locally Advanced and/or Positive Regional Lymph Node Upper Tract Urothelial Carcinoma. J Clin Oncol 2017;35:852-60.

41. Luo Y, Feng BF, Wei DC, et al. Prospective controlled observation ofeffect of adjuvant chemotherapy onsurvival and prognosis ofhigh-risk upper tract urothelial carcinoma patients underwent radical nephroureterectomy. Zhonghua Yi Xue Za Zhi 2019;99:3158-63.

42. Bellmunt J, Hussain M, Gschwend JE, et al. Adjuvant atezolizumab versus observation in muscle-invasive urothelial carcinoma (IMvigor010): a multicentre, open-label, randomised, phase 3 trial. Lancet Oncol 2021;22:525-37.

43. Powles T, Assaf ZJ, Davarpanah N, et al. 1O - Clinical outcomes in post-operative ctDNA-positive muscleinvasive urothelial carcinoma (MIUC) patients after atezolizumab adjuvant therapy. Ann Oncol 2020;31:S1417-S24.

44. Bajorin DF, Witjes JA, Gschwend JE, et al. Adjuvant Nivolumab versus Placebo in Muscle-Invasive Urothelial Carcinoma. N Engl J Med 2021;384:2102-14.

45. Sternberg CN, de Mulder PH, Schornagel JH, et al. Randomized phase III trial of high-dose-intensity methotrexate, vinblastine, doxorubicin, and cisplatin (MVAC) chemotherapy and recombinant human granulocyte colony-stimulating factor versus classic MVAC in advanced urothelial tract tumors: European Organization for Research and Treatment of Cancer Protocol no. 30924. J Clin Oncol 2001;19:2638-46.

46. von der Maase H, Sengelov L, Roberts JT, et al. Longterm survival results of a randomized trial comparing gemcitabine plus cisplatin, with methotrexate, vinblastine, doxorubicin, plus cisplatin in patients with bladder cancer. J Clin Oncol 2005;23:4602-8.

47. Galsky MD, Arija JÁA, Bamias A, et al. Atezolizumab with or without chemotherapy in metastatic urothelial cancer (IMvigor130): a multicentre, randomised, placebocontrolled phase 3 trial. Lancet 2020;395:1547-57.

48. Powles T, Csőszi T, Özgüroğlu M, et al. Pembrolizumab alone or combined with chemotherapy versus chemotherapy as first-line therapy for advanced urothelial carcinoma (KEYNOTE-361): a randomised, open-label, phase 3 trial. Lancet Oncol 2021;22:931-45.

49. Powles T, van der Heijden MS, Castellano D, et al. Durvalumab alone and durvalumab plus tremelimumab versus chemotherapy in previously untreated patients with unresectable, locally advanced or metastatic urothelial carcinoma (DANUBE): a randomised, open-label, multicentre, phase 3 trial. Lancet Oncol 2020;21:1574-88.

50. Moschini M, Shariat SF, Rouprêt M, et al. Impact of Primary Tumor Location on Survival from the European Organization for the Research and Treatment of Cancer Advanced Urothelial Cancer Studies. J Urol 
2018;199:1149-57.

51. Powles T, Park SH, Voog E, et al. Avelumab Maintenance Therapy for Advanced or Metastatic Urothelial Carcinoma. N Engl J Med 2020;383:1218-30.

52. Bellmunt J, Fougeray R, Rosenberg JE, et al. Longterm survival results of a randomized phase III trial of vinflunine plus best supportive care versus best supportive care alone in advanced urothelial carcinoma patients after failure of platinum-based chemotherapy. Ann Oncol 2013;24:1466-72.

53. Balar AV, Galsky MD, Rosenberg JE, et al. Atezolizumab as first-line treatment in cisplatin-ineligible patients with locally advanced and metastatic urothelial carcinoma: a single-arm, multicentre, phase 2 trial. Lancet 2017;389:67-76.

54. Balar AV, Castellano D, O'Donnell PH, et al. First-line pembrolizumab in cisplatin-ineligible patients with locally advanced and unresectable or metastatic urothelial cancer (KEYNOTE-052): a multicentre, single-arm, phase 2 study. Lancet Oncol 2017;18:1483-92.

55. Bellmunt J, de Wit R, Vaughn DJ, et al. Pembrolizumab as Second-Line Therapy for Advanced Urothelial Carcinoma. N Engl J Med 2017;376:1015-26.

56. Powles T, Durán I, van der Heijden MS, et al. Atezolizumab versus chemotherapy in patients with platinum-treated locally advanced or metastatic urothelial carcinoma (IMvigor211): a multicentre, open-label, phase 3 randomised controlled trial. Lancet 2018;391:748-57.

57. Sharma P, Retz M, Siefker-Radtke A, et al. Nivolumab in metastatic urothelial carcinoma after platinum therapy (CheckMate 275): a multicentre, single-arm, phase 2 trial. Lancet Oncol 2017;18:312-22.

58. Powles T, O'Donnell PH, Massard C, et al. Efficacy and Safety of Durvalumab in Locally Advanced or Metastatic Urothelial Carcinoma: Updated Results From a Phase 1/2 Open-label Study. JAMA Oncol 2017;3:e172411.

59. Apolo AB, Infante JR, Balmanoukian A, et al. Avelumab, an Anti-Programmed Death-Ligand 1 Antibody, In Patients With Refractory Metastatic Urothelial Carcinoma: Results From a Multicenter, Phase Ib Study. J Clin Oncol
2017;35:2117-24.

60. Robinson BD, Vlachostergios PJ, Bhinder B, et al. Upper tract urothelial carcinoma has a luminal-papillary T-cell depleted contexture and activated FGFR3 signaling. Nat Commun 2019;10:2977.

61. Mollica V, Rizzo A, Montironi R, et al. Current Strategies and Novel Therapeutic Approaches for Metastatic Urothelial Carcinoma. Cancers (Basel) 2020;12:1449.

62. Loriot Y, Necchi A, Park SH, et al. Erdafitinib in Locally Advanced or Metastatic Urothelial Carcinoma. N Engl J Med 2019;381:338-48.

63. Rosenberg J, Sridhar SS, Zhang J, et al. EV-101: A Phase I Study of Single-Agent Enfortumab Vedotin in Patients With Nectin-4-Positive Solid Tumors, Including Metastatic Urothelial Carcinoma. J Clin Oncol 2020;38:1041-9.

64. O'Donnell P, Galsky MD, Rosenberg JE, et al. 746P - EV-201: Long-term results of enfortumab vedotin monotherapy for locally advanced or metastatic urothelial cancer previously treated with platinum and PD-1/PD-L1 inhibitors. Ann Oncol 2020;31:S579-S580.

65. Powles T, Rosenberg JE, Sonpavde GP, et al. Enfortumab Vedotin in Previously Treated Advanced Urothelial Carcinoma. N Engl J Med 2021;384:1125-35.

66. Rosenberg JE, Flaig TW, Friedlander TW, et al. Study EV-103: Durability results of enfortumab vedotin plus pembrolizumab for locally advanced or metastatic urothelial carcinoma. J Clin Oncol 2020;38:abstr 441.

67. Hassler MR, Bray F, Catto JWF, et al. Molecular Characterization of Upper Tract Urothelial Carcinoma in the Era of Next-generation Sequencing: A Systematic Review of the Current Literature. Eur Urol 2020;78:209-20.

68. Necchi A, Madison R, Pal SK, et al. Comprehensive Genomic Profiling of Upper-tract and Bladder Urothelial Carcinoma. Eur Urol Focus 2020. [Epub ahead of print]. doi: 10.1016/j.euf.2020.08.001.

69. Yoshida T, Kates M, Fujita K, et al. Predictive biomarkers for drug response in bladder cancer. Int J Urol 2019;26:1044-53.
Cite this article as: Gust KM, Resch I, D'Andrea D, Shariat SF. Update on systemic treatment of upper tract urothelial carcinoma: a narrative review of the literature. Transl Androl Urol 2021;10(10):4051-4061. doi: 10.21037/tau-21-47 